\title{
Preparing for the delivery of PrEP: Lessons from the implementation of integrated reproductive health and HIV services in Kenya
}

Emily McDonald Evens ( $\nabla$ eevens@fhi360.org )

FHI 360 https://orcid.org/0000-0002-7369-458X

Theresa Hoke

FHI 360

Margaret Eichleay

FHI 360

\section{Patrick Olsen}

Industry Standard Research

Alice Olawo

FHI 360

George Githuka

Kenya Ministry of Health

Research

Keywords: Pre-exposure prophylaxis, Kenya, Integrated health, HIV

Posted Date: January 20th, 2020

DOI: https://doi.org/10.21203/rs.2.21345/v1

License: (c) (i) This work is licensed under a Creative Commons Attribution 4.0 International License.

Read Full License 


\section{Abstract}

Background: In 2015, the WHO issued guidelines recommending oral pre-exposure prophylaxis (PrEP) for individuals at substantial risk of HIV. Given their role in health service delivery, ministries of health are assuming major responsibility for PrEP services in sub-Saharan Africa. However, these are often strained and under-resourced. We measured the delivery of selected integrated services and identified the factors that impede effective delivery of integrated services to prepare for the integration of PrEP into health services for women.

Methods: Three services were examined: 1) HIV testing within family planning, 2) HIV testing within general outpatient services, and 3) care and prevention services for HIV-positive clients not eligible for antiretrovirals ("Pre-ART"). Descriptive, mixed-methods research was conducted in three Kenyan counties using: service delivery observations $(n=3246)$ and client exit interviews $(n=3257)$ in public facilities, and semi-structured interviews with providers, managers and officials $(n=112)$.

Results: Integrated services were not consistently implemented. HIV testing was not systematically offered or received; most clients were not aware testing was available, and tests were largely unavailable. Pre-ART services did not systematically deliver mandated services. Rates of cervical cancer and tuberculosis screening were especially low; a concerning gap given the elevated risk for HIV-positive women. Supply-side challenges of staffing, infrastructure and commodity shortages were common.

Conclusions: In an era of significant demands on treatment resources and decreasing funding for prevention, the identification of the technical, material, and human resources required for successful and sustainable delivery of integrated services must be addressed to support successful PrEP implementation.

Key words: Pre-exposure prophylaxis, Kenya, Integrated health, HIV

\section{Background}

Scientists have made great strides over the past two decades producing biomedical interventions using antiretroviral drugs to prevent acquisition of HIV (1). A body of research has established the safety and effectiveness of tenofovir (tenofovir disoproxil fumarate (TDF)) taken orally to prevent HIV acquisition among consistent users (2). The World Health Organization (WHO) issued guidance in 2015 endorsing provision of oral pre-exposure prophylaxis (PrEP) to populations at substantial risk of HIV, defined as those with incidence of $3 \%$ or higher (3). Responding to WHO guidance, ministries of health in subSaharan African countries with a high HIV burden have begun delivering PrEP to populations most at risk of HIV. A recent report reveals, however, that countries have been slow in achieving levels of PrEP use needed to achieve a marked public health impact (4). Low coverage is in part attributed to an inadequate delivery platform rooted in health system weaknesses (5). Evidence is needed on the nature of these service delivery shortcomings to guide remedial action. 
Public sector health facilities in sub-Saharan Africa deserve close examination given their predominant role in PrEP rollout. Adding PrEP delivery to the services offered by facilities that are already underresourced and overly strained could result in quality deficiencies or bottlenecks (6-9). This is especially a risk given how PrEP delivery requires a package of services including the following:

1. Publicizing services and screening clients to identify those at elevated risk for HIV

2. Dispensing medication and counseling clients about correct use, including adherence, (14)

3. Counseling clients on behavioral risk reduction (8)

4. Providing periodic HIV testing and toxicity screening (8)

5. Tracking clients and providing support to encourage retention in services

While trials testing PrEP have been implemented in highly controlled and well-resourced research contexts, there is still limited documented experience to guide the design of PrEP services in non-research settings $(5,15-17)$. However, because PrEP is being added to existing health services as opposed to only being offered as a stand-alone vertical program (12), its introduction can be considered another form of integrated service delivery $(18,19)$. Examining the provision of other types of integrated health services can therefore provide useful lessons. Offering multiple health services in a single clinical encounter produces efficiencies for providers and client alike $(17,20)$, but integrated service delivery also presents challenges (21). Research has revealed that providers may miss opportunities to provide multiple sexual and reproductive health services when services are supposed to be integrated, and these lapses have been attributed to health system limitations and overwhelming demands placed on providers $(22,23)$. Decisions about how and where PrEP will be introduced in resource-poor health systems should be guided by evidence showing the factors favoring and impeding successful health service integration (8).

We conducted a descriptive, mixed-methods research study in Kenya, where the Ministry of Health was implementing The Minimum Package for Reproductive Health/HIV Integrated Services (24). Aimed at operationalizing the National RH-HIV Integration Strategy within resource-constrained settings, the Minimum Package was a set of recommendations for integrating all types of health services. Facilities implementing the Minimum Package were expected to offer multiple diagnostic, treatment, promotion, and preventive services to clients in a single visit. Examining implementation of selected components of 
the Minimum Package provided a means of exploring potential challenges that could be anticipated with PrEP service delivery. The objective was to examine the extent to which selected integrated RH/HIVrelated services were currently being delivered as intended in these facilities. This study focused on women because at the time of study development, topical microbicides used vaginally were the most promising forms of pre-exposure prophylaxis. Study results were intended to inform the introduction of the broad class of ARV-based PrEP products in public sector facilities in resource-constrained settings (13).

\section{Methods}

We chose Minimum Package components to study based on whether they: 1) involved health service delivery functions similar to those required for PrEP services; 2) served female clients with demographic profiles similar to those of clients likely to be targeted for PrEP; 3) were operational as an integrated service in the three participating counties; and 4) involved types of health services that are potential base services for the incorporation of PrEP delivery.

The selection of these services was informed by a previous study that investigated which service settings would be best to deliver topical, oral, and injectable PrEP to at-risk groups. $(25,26)$ Findings showed that clients would prefer to receive PrEP products in locations that they already visit for healthcare. Respondents also stressed the importance of having PrEP products available in multiple settings to increase access, support adherence, and address concerns over privacy, convenience, and quality of care. The three integrated services we examined are: HIV testing within family planning services, HIV testing within general outpatient services, and HIV care for clients not yet eligible for ART within Comprehensive Care Centers (CCCs) which provide care for people living with HIV.

HIV testing services (HTS) offered within family planning and general outpatient services. According to the Minimum Package, HTS should be integrated into family planning (FP) and general outpatient department (OPD) services, with referrals of HIV-positive clients to HIV/AIDS services. This form of service integration is relevant to PrEP service delivery, given that HIV testing to confirm seronegative status is an essential first step for PrEP services. Additionally, previous research has indicated that FP and OPD services are viewed by potential users of PrEP as possible venues for PrEP delivery given that they serve high volumes of sexually active women in a context relatively free of stigma $(8,27)$. Both FP and OPD services have been proposed as a potential base service to which PrEP could be added $(26,28$ $31)$.

Provision of HIV care and prevention services to clients who are not yet eligible for ART (pre-ART services) within Comprehensive Care Centers (CCCs). The Minimum Package calls for the provision of screening and testing for sexually transmitted infections (STIs), malaria, and tuberculosis, screening for reproductive organ cancer, family planning counseling and provision, education on nutrition and sanitation, and psychosocial support, all as part of HIV/AIDS services. Similar to PrEP services, these services require multiple service delivery functions including: screening for need, dispensing 
pharmaceuticals, counseling on correct use, and tracking clients over time. Service delivery settings caring for HIV-positive individuals have been commonly discussed as a potential delivery channel for offering integrated services (8). Although clients living with HIV clearly are not candidates for PrEP, we chose to assess HIV service settings' demonstrated capacity to deliver the full package of pre-ART care as an indication of their readiness to deliver an additional service (i.e., PrEP).

\section{Data collection}

The study was conducted in Nairobi, Mombasa, and Kisumu counties, which represent Kenya's largest urban populations (32) and some of the country's highest rates of HIV prevalence ( 8\%, 11\%, and 19\% respectively) (33). Data collection methods included observation of service delivery, exit interviews, and semi-structured interviews with key informants (see Table 1). Data on the receipt of services delineated in the Minimum Package for clients was gathered from client exit interviews and observations. Data collectors were male and female community members, fluent in both English and the primary local language in the study area-either Kiswahili or Dholuo. All data collectors had previous experience in data collection and received additional training in research ethics and research methods by the research team.

\section{Table 1: Sample size by service and county}

\begin{tabular}{|c|c|c|c|c|c|c|c|}
\hline \multirow[b]{2}{*}{$\begin{array}{c}\text { Site } \\
\text { (\# facilities) }\end{array}$} & \multicolumn{2}{|c|}{ Family Planning } & \multicolumn{2}{|c|}{ Outpatient } & \multicolumn{2}{|c|}{ Pre-ART } & \multirow{2}{*}{$\begin{array}{c}\text { Key Informant } \\
\text { interviews }\end{array}$} \\
\hline & Observations & $\begin{array}{c}\text { Exit } \\
\text { Interviews }\end{array}$ & Observations & $\begin{array}{c}\text { Exit } \\
\text { Interviews }\end{array}$ & Observations & $\begin{array}{c}\text { Exit } \\
\text { Interviews }\end{array}$ & \\
\hline Nairobi (15) & 485 & 482 & 500 & 510 & 195 & 197 & 30 \\
\hline Kisumu (15) & 361 & 384 & 331 & 330 & 267 & 262 & 33 \\
\hline Mombasa (15) & 497 & 483 & 483 & 482 & 127 & 127 & 32 \\
\hline County/National & -- & -- & -- & -- & -- & -- & 18 \\
\hline Total & 1343 & 1349 & 1314 & 1322 & 589 & 586 & 113 \\
\hline
\end{tabular}

\section{Observations}

Observations of individual, client-provider interactions were conducted in 45 facilities offering integrated services. These facilities were selected in consultation with decision-makers involved in developing Kenya's policies and planning for eventual PrEP delivery. Selected sites had 1) high client flow, 2) provided the three selected integrated services, and 3) were not receiving substantial donor funds for general operations according to local decisionmakers. One provider in each service was observed each day; if there was more than one provider in the service on the day of observation then the provider seeing the greatest number of clients was selected. Using a checklist, research assistants (RAs) recorded service content to assess the consistency with which the service was offered and the extent to which provider performance conformed to service delivery guidelines. Group counseling, if available, was not observed. 
Research assistants conducting observations had prior clinical training or were highly experienced in conducting data collection for health services research studies and extensively trained to conduct observations.

\section{Client Exit Interviews}

In exit interviews, clients were asked about their experience on the day of the interview. For clients in CCCs, this was expanded to include whether they had received specified services from a provider at that facility since learning their positive HIV status. Structured client exit interviews were conducted in the facilities where observations occurred; approximately $85 \%$ of observed clients also participated in the exit interview. Interview questions explored clients' need for the integrated services, whether they received those services, and their knowledge about follow-up actions they should take. Participants for client exit interviews were recruited from waiting areas for the selected services at each facility prior to their interactions with providers. Women were eligible if they were of reproductive age (18 - 49 years), seeking services for themselves, and spoke either Swahili, Dholuo, or English. Client exit interviews were conducted in each participant's preferred language in a private setting within the facility.

\section{Key Informant Interviews}

Semi-structured key informant interviews, including closed- and open-ended questions were conducted with managers and providers at the 15 integrated facilities, as well as managers working in 9 nonintegrated facilities to explore the factors supporting and impeding implementation of the Minimum Package. Additionally, interviews were conducted with 18 county-level and national-level officials to trace the root causes of barriers and facilitators encountered at the facility level (see Table 1). All key informant interviews were conducted in English, audio-recorded and transcribed.

\section{Analysis methods:}

Quantitative data from observations, client exit interviews and the closed-ended portions of the key informant interviews were collected electronically on password-protected Android tablet computers using Pendragon software v.7 and analyzed using SAS v.9. $2^{\circledR}$ and Stata v.12. No statistical comparisons were made between types of clients, as this was not the intent of the study.

All qualitative data from key informant interviews were structurally coded according to question and answer using a data reduction matrix. First, the research team read all transcripts for quality and clarity and corrected any errors or unclear segments. Then, answers to each specific question were categorized using either discrete categories or short summaries and associated quotes, as appropriate. After coding the first few transcripts the coders compared application of codes and reconciled any discrepancies on how codes were applied. An inductive, thematic analysis was then conducted using memo writing to answer the research questions. 
Representatives from county- and national-level government were involved in the design and interpretation of study data from the inception of the study to the development of the final products. At the conclusion of the study, a data interpretation workshop was held with governmental stakeholders and their partners who were responsible for the delivery of integrated sexual and reproductive health services and preparation for PrEP delivery in Kenya to review and prioritize study results.

\section{Ethics}

The study was approved by the institutional review boards at FHI 360 and the Kenya Medical Research Institute (KEMRI), and all research was conducted in full compliance with the approved study protocol. Each participant provided his or her verbal informed consent prior to clinical observations and exitinterviews. Verbal consent was sought because: 1) the study was minimal risk; 2) the data collection instruments did not require disclosure of identifying information; and 3) a written signature would provide the only link between the participant and the study. RAs documented consent by signing and dating the informed consent forms after obtaining verbal consent from each participant.

\section{Results}

\section{Client profile}

Family planning clients were an average of 26 years old and mostly married (88\%); outpatient clients were an average of 28 years and less than three-quarters were married (70\%). Clients waiting to start ART were the oldest with an average of 30 years; they had been living with HIV for an average of 2.7 years and two-thirds (66\%) were married.

\section{Delivery of HTS within Family Planning and Outpatient Services}

According to client exit interviews, nearly all clients had been tested for HIV previously (Figure 1). Eleven percent of FP clients and $9 \%$ of OPD clients were tested on the day of the observation. Over half of clients reported being tested in the last six months (58\% FP, 54\% OPD); about one-third reported being tested more than six months ago (29\% FP, 33\% OPD). A very small number of clients reported never having received testing ( $1 \% \mathrm{FP}, 4 \% \mathrm{OPD})$.

\section{Figure 1. Time since last HIV test, by client type, from client exit interviews}

The reasons most clients did not get tested on the day of the interview included: already knowing their status ( $41 \% \mathrm{FP}, 37 \%$ OPD), visiting the facility for some other purpose (39\% FP, 39\% OPD), and providers 
not offering the test (32\% FP, 34\% OPD) (data not shown).

Data on the delivery of HTS from both client exit interviews and observations are reported in Table 2. The intention of reporting both sets of results is to illustrate the relative frequency with which clients receive services rather than to directly compare the two. Just over one-third of family planning (38\%) and outpatient (35\%) clients knew that testing was available at the facility on the day of the interview. (This information was not available from observation data as RAs did not ask clients questions during the observations.) In the exit interviews, about one-third (34\%) of FP clients and one-quarter (25\%) of OPD clients reported that the provider mentioned getting tested for HIV on the day of the interview; however, according to observations of provider-client interactions, providers mentioned HIV testing much less.

\section{Table 2: HTS received, by client type, as reported in exit interviews and observed}

\begin{tabular}{lcccc}
\hline & \multicolumn{2}{c}{ Family Planning \% } & \multicolumn{2}{c}{ Outpatient \% } \\
& Exit & Obs. & Exit & Obs. \\
& $(\mathrm{n}=1349)$ & $(\mathrm{n}=1343)$ & $(\mathrm{n}=1322)$ & $(\mathrm{n}=1314)$ \\
Knew HTS available & 37.8 & $\mathrm{NA}$ & 34.7 & $\mathrm{NA}$ \\
Provider mentioned HIV testing today & 34.1 & 12.0 & 25.4 & 9.2 \\
Knew HTS available & & & 34.7 & $\mathrm{NA}$ \\
Counseled on HIV prevention* & 37.8 & $\mathrm{NA}$ & 14.1 & 8.7 \\
Received condoms & 21.9 & 19.3 & 1.4 & 0.2 \\
\hline
\end{tabular}

Obs.: Client-provider observations

NA: Data not available

*For exit interviews, HIV prevention counseling was measured by clients saying if the provider "mentioned ways of preventing HIV infection." In the observations, HIV prevention counseling was counted if the provider mentioned any of the following: condoms as prevention, benefit of knowing status, couples testing, the HIV test process, or HIV disclosure.

HIV prevention counseling was infrequently provided to both FP and OPD clients. Only $22 \%$ of FP clients and $14 \%$ of OPD clients said their providers gave them prevention messages. (Table 2) Similarly, low proportions of clients received these messages during observed consultations. (Table 2) Furthermore, over $50 \%$ of those who reported having been counseled on HIV prevention could not provide any details on the content of the counseling (data not shown) including information on abstinence, having only one partner, using condoms and avoiding needles as methods to prevent HIV. Most facilities were observed to have condoms available during observations; however, a small number of facilities ( 2 of 18$)$ had condoms available during fewer than $20 \%$ of the observations. Very few clients from either service reported receiving condoms at the facility.

Among the 270 clients who were tested for HIV on the day of the interview, $56 \%$ of clients exiting both family planning and outpatient services said they had received counseling on ways to prevent HIV transmission, such as condom use and monogamy (data not shown). Among the 64 family planning 
clients whose HIV testing was observed for this study, approximately half were counseled on the benefits of knowing their status and on couples testing. (Table 3) A greater proportion (70\%) had the testing process explained to them and $75 \%$ were observed to give their consent for the test.

\section{Table 3. Frequency of components of HIV counseling according to Minimum Package guidelines, among family planning clients* whose test process was observed}

\begin{tabular}{lc}
\hline & $\begin{array}{c}\text { Family planning \% } \\
(\mathrm{n}=64)\end{array}$ \\
\hline Provider tells client benefit of knowing status & 54.7 \\
Provider tells client about couples testing & 51.6 \\
Provider explains HIV test process & 70.3 \\
\hline Provider gets client's consent for test & 75.0 \\
\hline
\end{tabular}

* This table reflects data from family planning clients only because we observed only 9HIV tests among outpatient clients.

Key informants shared information on the approach they take in offering HIV testing. Providers commonly described making decisions as to which clients to offer HIV testing based on a clients' appearance and clinical symptoms; specifically, those who presented with signs and symptoms of HIV or other STIs, new mothers, those with opportunistic infections, or those who generally appeared weak were mentioned as groups to whom HIV testing was offered. As one outpatient provider noted,

"I will just look at the patient's condition and suspect the patient is having HIV or I have treated this patient for a long time, but they are not responding so I just send the client for DTC." [NOTE: diagnostic testing and counseling, or DTC, targets clients with HIV-related signs and symptoms]."

- Provider, Family Planning department

Some providers reported that risk of HIV was not readily identifiable. One FP provider stated that, "... when it comes to HIV, it doesn't have the boundaries, anyone can have it."However, more key informants identified particular social characteristics as important for determining risk. Across providers and managers in both FP and OPD services, respondents identified young, sexually active, single women; fishermen; truck drivers' wives; commercial sex workers; and IV drug users as high-risk groups.

Interviews also illuminated service delivery challenges that could limit integration of HTS into the two services. An inconsistent supply of test kits was a commonly cited limitation to the provision of HTS during FP and OPD services. For $38 \%$ of family planning visits and $45 \%$ of outpatient visits observed, RAs documented that HIV test kits were not available for the client (data not shown). This resulted in facilities having to refer clients to other facilities. In addition, family planning providers and managers reported a lack of basic family planning commodities, including injectable contraceptive and long-acting methods, as a regular and persistent problem at the facility level. 
“...Now we are facing shortage of test kits. They (clients) are not being tested, they are being referred to another facility unless [except for] the one you really, really want to do, but those who are coming for voluntary testing we are not doing."

- Provider, Outpatient Department

\section{Pre-ART}

Client exit interviews, observations and key informant interviews, show similar patterns of inconsistent provision of the full range of pre-ART services (See Table 4). During the visit on the day of the exit interview, the most commonly received service was the receipt of co-trimoxazole, an antibiotic given prophylactically to HIV-positive individuals to prevent common infections. Nutrition, HIV prevention, and family planning were the next most commonly received services, with approximately one-third of providers discussing appetite with clients and nearly half discussing family planning and HIV protection for partners. Rates of TB prevention counseling (10\%), testing for cervical cancer (5\%), and TB testing (3\%) on the day of the interview were particularly low. However, nearly half $(47 \%)$ of pre-ART clients reported that a provider in the facility had mentioned tuberculosis prevention since the client learned their HIV status. Among women who self-reported having a cough for at least 3 weeks or who had been coughing up blood, only $38 \%$ had been tested for TB at the facility since learning their status. (Data not shown). Aside from the receipt of co-trimoxazole, which in some cases was dispensed from a pharmacy and not during the observed provider examination, results from the observations of client-provider interactions regarding the services received were fairly similar to those reported by clients.

Table 4. Services received by pre-ART clients, self-reported and in observations, by timing of receipt 


\begin{tabular}{|c|c|c|c|c|}
\hline \multicolumn{2}{|c|}{ Counseling/Service topic } & \multirow{2}{*}{$\begin{array}{c}\text { \% ever received } \\
97.8\end{array}$} & \multirow{2}{*}{$\begin{array}{c}\text { \% received today } \\
93.0\end{array}$} & \multirow{2}{*}{$\begin{array}{c}\text { \% received today } \\
53.5\end{array}$} \\
\hline Infectious & Co-trimoxazole provided & & & \\
\hline & Genital pain/discomfort discussed & 40.4 & 13.8 & 7.8 \\
\hline & Malaria prevention (bed nets, insect spray) & 63.5 & 10.6 & 9.0 \\
\hline & TB prevention discussed & 47.4 & 9.6 & 9.0 \\
\hline & Cervical cancer screening & 26.6 & 4.6 & 1.7 \\
\hline & Malaria medicine provided & 24.9 & 4.3 & 1.7 \\
\hline & TB test & 21.2 & 3.2 & 4.2 \\
\hline & STI test & 20.8 & 1.7 & 0.2 \\
\hline & Vaccination provided & 7.0 & 0.2 & 0 \\
\hline \multirow[t]{3}{*}{ Nutrition } & Appetite discussed & 58.0 & 30.9 & 29.0 \\
\hline & Nutritional supplements/vitamins provided & 39.6 & 15.4 & 11.0 \\
\hline & Food assistance programs discussed & 16.6 & 5.0 & 4.8 \\
\hline \multirow[t]{2}{*}{ HIV prevention } & Protecting sexual partner from HIV discussed & 90.4 & 42.8 & 49.9 \\
\hline & Partner testing mentioned & 88.1 & 36.7 & 21.7 \\
\hline \multirow[t]{4}{*}{ Pregnancy } & Family planning discussed & 79.4 & 41.8 & 52.1 \\
\hline & Safe pregnancy discussed & 72.0 & 17.6 & 5.4 \\
\hline & Pregnancy desire discussed & 46.6 & 17.4 & 12.1 \\
\hline & FP method provided & 57.2 & 9.9 & 2.0 \\
\hline \multirow[t]{2}{*}{ Psycho-social } & Disclosing status discussed & 67.4 & 17.1 & 34.1 \\
\hline & Joining HIV support group discussed & 56.1 & 14.7 & 28.7 \\
\hline \multirow[t]{2}{*}{ Sanitation } & Safe drinking water education & 74.4 & 17.6 & 13.4 \\
\hline & Hand-washing education & 68.9 & 13.3 & 7.0 \\
\hline
\end{tabular}

Key informants were asked to explain how they decided which services a pre-ART client should receive. Some respondents recognized the need to provide services universally. One provider in a CCC stated: "If a client comes here I have to screen for TB because I have checklist for checking... they come in I have to go through the TB tool, STI tool, all those..." Many other providers and managers, however, described how clients presenting with opportunistic infections or signs and symptoms of HIV, clients who are pregnant, and clients who have TB or a low CD4 count, are given priority in the form of being seen immediately or given more time with a provider. Respondents also discussed tailoring services they provided to CCC clients according to social characteristics, allowing them to broaden the services delivered to clients. Clients perceived to be part of high-risk populations, such as commercial sex workers, drug users, men who have sex with men, alcoholics, and selected occupational groups such as long-distance truck drivers or fisherman, often received different services. 


\section{Health system factors facilitating Minimum Package rollout}

County and national key informants expressed positive attitudes towards the provision of integrated services conducive to the provision of the Minimum Package. These participants described multiple benefits of integration including: time savings for clients and providers, the opportunity to capture more clients, increased client satisfaction, and elimination of the need for clients to wait in more than one line. A few respondents also stated that integrated services would increase client compliance and save resources.

"That (integration) is the only way to go if you are to catch up with HIV...you have to have an interest in family planning which is very key especially now that we want to do elimination of mother to child transmission... that is the only way we should go...shuffling patients from left to right they even get lost for follow up... so we have to integrate our services under one roof."

- County level health official

Respondents also positively described the support they received from the county and national level government and external partners in the form of trainings and supportive supervision to increase provider capacity to deliver integrated services, technical input to help identify and make improvements to address facility weaknesses, and assistance with organizing community outreach activities. Assistance from external partners to hire additional staff, make infrastructure improvements and procurement were additional areas of support cited.

"There have been a lot of trainings, especially to the health care givers, and much of it is towards HTS... our counselors and nurses have been empowered so much. There is adherence training...there is [sic] people living positively training, there is disclosure training... There is a way of giving out benefits of carrying out HTS, so they have also been empowered with that technique. These ones actually enhance our uptake on providing HTS."

- Provider, Outpatient Department

\section{Health system constraints impeding Minimum Package rollout}

Results from key informant interviews provide insight into the health systems-level factors that impede effective delivery of integrated services. At the most basic level, knowledge of the Minimum Package was far from universal, even in the integrated facilities. Only 24 of 64 facility-level managers had heard of these service delivery guidelines before the interview. Among all respondents, county- and national-level managers were more apt to be aware of the Minimum Package compared to managers and providers working in health facilities. 
Key informants also shared information about weaknesses in the health system that constrained implementation of the Minimum Package more generally. The vast majority of key informants at all levels spontaneously mentioned that staff shortages were a systemic problem at the facility level. One national-level key informant stated:

"I think resource allocation...especially the staff [is a key challenge], when you look at the shortage they have, there is shortage across board and every cadre, for clinical officers... we need 5,700 more...in nurses they are talking about another 10,000... The issue of shortage is very critical so for integration we really need to look at the human resource aspect."

- National Level Official

Key informants reported that staff shortages often left providers overburdened with clients and resulted in the need to prioritize certain clients for HTS.

"Specifically,... HTS is for all clients, but due to the fact that you know there is problem with the manpower, the staffs are few, I think what we normally do here is to request patients who have major presentation of HIVIAIDS to go for, we provide those clients, we also ask those who are high risk-the commercial sex workers, people with multiple partners, STIs and rape cases those kind of patients we normally ask them to go for VCT."

- Manager, Outpatient department

Other staffing issues included situations where one staff member had responsibility for all clinical services, as one CCC manager stated, "I am the one doing the pharmacy, I am the one doing the blood, I am the one...you know, you do everything." Additionally, the overall lack of supervision or support for providers and managers was commonly cited.

"When staff are over worked then they look for shortcuts or when nobody gives you a pat on your back for job well done then short cuts are taken to make sure that the queue of clients is short, you evade some things to favor yourself... you say, what the hell, I will not take temperature or height or weight. That is human...but, it can be controlled and it's only that they don't want to support us (providers). That is why some of these things will go on for years and years."

- Provider, CCC department, Kisumu County

Inadequate staff training was raised by key informants at every level of the health system and in each type of service explored in the study. Insufficient resources allocated to health facility staff development led to providers not receiving in-service training for some services they were expected to deliver.

"I have not received any family planning training [since college] 15 years ago so what I have learnt in family planning is my own initiative. I read books and the family planning packages book on my own to be able to provide family planning services to the clients." 
- Provider, Family Planning department

Health facilities were found to frequently rely on volunteers for the provision of services, especially for HTS. Reliance on unpaid workers is a severe challenge to the sustained provision of health services, particularly given the lack of incentives for volunteers to work on a regular basis.

Key informants reported that the limitations described above were key contributors to longer client wait times that obstructed the provision of integrated services. This point was reinforced by exit interviews, with about one-third of family planning and pre-ART clients and half of outpatients saying that the wait time to see the provider was too long.

Key informants also reported a general lack of privacy and inadequate infrastructure in facilities that made it difficult for health facility staff to deliver services and for clients to receive high-quality care. This was especially true regarding space for HIV counseling and the provision of sexual reproductive health services such as cervical cancer screening or IUD insertion. Key informants reported that there is often only one room for multiple clients, creating challenges for maintaining privacy and confidentiality.

"In this facility... there is no privacy because it is one room where the clinicians and the counselor sit. So, sometimes a clinician has a client that needs to be examined on the couch and that couch is next to where the counselor sits... and so there is lack of privacy because the counselor plus her client will be hearing what I am telling the patient I am examining. At the same time, we will be able to hear what the counselor and the client are talking about. So... that will also hinder the HTS."

- Provider, Outpatient department, Kisumu County

Key informants also noted demand-side challenges specific to the provision of HIV-related services. Specifically, many described lack of attendance for pre-ART services as strongly linked to the stigma associated with seeking care from a facility offering HIV services.

"The largest challenge is stigma. Some new clients aren't comfortable with sitting in the area that is known as the CCC client area. Clients don't want to be seen coming to the clinic."

- Provider, CCC department, Nairobi County

\section{Work with in-country stakeholders}

Stakeholders acknowledged that the health system weaknesses identified in the study were factors preventing delivery of the Minimum Package, and they offered alternative perspectives on many of the issues highlighted by study results. For example, stakeholders discussed how issues related to commodities, such as a lack of HIV test kits, required attention in addition to supply. They suggested a focus on identifying root causes of test kit stock-outs, such as theft, under-reporting of testing or inaccurate recordkeeping, and limitations in forecasting made by providers at the facility and county 
levels. Issues related to training could be partially addressed with improved tracking of the training needs and capacity of staff.

\section{Discussion}

This examination of the implementation of Kenya's Minimum Package of integrated RH/HIV services revealed what can happen when busy public-sector health services are tasked with new responsibilities. We found that integrated services were not being consistently implemented within FP and OPD services. HIV testing was not systematically offered to FP and OPD clients, and very few clients actually received testing. Most clients were not aware that HIV testing was available in the facility where they received services. Similarly, providers did not systematically provide HIV-prevention counseling. Observations revealed that HIV tests were unavailable for large numbers of clients; this shortage means that providers cannot encourage clients to be tested the day of their visit and that clients who come specifically to be tested may encounter problems. With the high proportion of clients reporting they had been tested in the past 6 months, one might conclude that these omissions in services are generally not problematic. However, the accuracy of clients' self-reports on timing of last HIV test is unknown. Furthermore, in highrisk settings such as these, communicating the availability of testing and providing prevention counseling and condom provision should be at near universal levels.

Our assessment of the services offered to pre-ART clients indicates that CCCs may not be well prepared to take on the added responsibility of PrEP service provision. Pre-ART services did not systematically deliver the range of services mandated in the Minimum Package to their clients. While the receipt of cotrimoxazole was high, less than half of clients reported receiving preventive services including discussions of HIV protection for partners or family planning. Especially low were the rates of testing for cervical cancer, TB prevention counseling and testing. These gaps are especially noteworthy given that HIV-positive women are at elevated risk for both cervical cancer and tuberculosis and are prioritized for these services according to national guidelines. Providers acknowledged stigma surrounding CCC services, a finding consistent with speculation by others, that clients may not be comfortable seeking prevention services in the same facility where others receive HIV/AIDS care and treatment. (8)

On a more promising note, rollout of the Minimum Package highlights both a willingness to address persistent and sensitive challenges. Study results documenting positive attitudes from providers, managers, and county and national-level officials towards the provision of integrated services highlights their commitment to addressing the full range of clients' needs. This is similar to qualitative research on providers' experiences with providing integrated services in Kenya that found providers value increasing their skills, variety and challenge in their work. Additionally, increasing client and personal job satisfaction is associated with providing integrated services. (34)

However, our data also demonstrate the considerable-and familiar-challenges faced by the delivery of the Minimum Package of integrated RH-HIV services. Service delivery is constrained by health system weaknesses such as inadequate infrastructure and inconsistent commodities and supplies. Challenges 
with training and supervision manifest in problems like limited awareness of service delivery requirements and inconsistent compliance even when guidelines are known. Sheer shortages of health personnel result in heavy workloads for providers and long waiting times for clients. Addressing staffing, supervision and training shortages is essential but comes with cost and sustainability implications that are also challenging. These issues have also been identified by other examinations of integrated services and in the limited body of knowledge addressing the introduction of PrEP. $(5,8,34)$ Qualitative data illustrate the prioritization providers and managers use within the constraints of the health system; those clients with obvious need often get the required services while asymptomatic clients often get passed by due to resource limitations.

These results have clear implications for the provision of oral PrEP. Service rollout will place similar burdens on the service delivery system, as they are being integrated into existing services and not standalone care (19). This study documented clear problems delivering services that are already supposed to be integrated, such as systematic offering of HIV testing in family planning services. This failure highlights the considerable challenges facing integrated RH/HIV services now and indicates the challenges that providers and health systems must face before they are ready to take on the added responsibilities of providing PrEP. Given the importance of knowing that a person is and remains HIV negative while taking PrEP, the lack of test kits will be another major barrier to PrEP provision. In resourcepoor settings facing similar circumstances, successful provision of PrEP will first require strengthening of systems for commodity management, provider training and supervision and reporting. Client management will involve a set of essential clinical steps, including intensive communication to ensure clients understand risks, use products effectively and grasp the importance of returning to facilities for repeated follow-up visits. Counseling skills, therefore, should be strengthened for services already provided before introducing PrEP, and health workforce solutions should be sought to help facilities prepare to take on additional time-consuming service delivery tasks.

To respond to these challenges, study findings informed the development of a PrEP Readiness Assessment Tool (https://www.fhi360.org/resource/microbicides-readiness-assessment-tool/, designed to diagnose and plan for the introduction of PrEP in public-sector facilities within developing countries. (35) The tool evaluates the extent to which the anticipated material, technical and human resources necessary for the implementation of PrEP are in place within a given facility. The tool is intended for immediate use, prior to the widespread availability and routine use of PrEP in service delivery settings. In doing so, it will help identify the service delivery and health systems reinforcements needed to prepare for PrEP delivery in the facility and what is needed to strengthen the provision of integrated services. The tool is organized into five domains that correspond with the World Health Organization (WHO) Health Systems Building Blocks (36) and the results from this study: health workforce, essential medicines, service delivery, information and research, and leadership and governance. Health financing is not included as a domain since this tool focuses at the facility level and facilities typically have limited control over health financing decision-making. Use of the tool at the facility level, with discussion of the results at the county and national level, may help facilities begin preparations for PrEP delivery. 
This study's limitations are noted. Foremost, this research was conducted prior to release of WHO guidelines supporting PrEP delivery and the launch of services in Kenya. In 2017 the government of Kenya launched PrEP delivery in selected health facilities nationwide, with 43,500 clients receiving PrEP services as of July 2019 (4). In the absence of systematic examination of PrEP service delivery in realworld settings in sub-Saharan Africa, the evidence produced by this study is relevant and informative. Another limitation is potential bias in recorded observations of client-provider interactions. While not expected to be significant, providers could alter their behavior during consultations that are observed, potentially biasing study results compared to unobserved interactions. Services received during observations could also be affected due to services clients have received previously; this study did not examine client records to determine whether clients were eligible or due for individual services.

Finally, reported data on the delivery of services from client exit interviews and observations differs. Neither type of data is assumed to provide a fully accurate report of service provision, and the two may differ due to recall or social desirability biases. Additionally, as RAs were located within the primary service delivery location, they may have failed to observe all the services-such as those in a stand-alone voluntary testing and counseling service, group counseling sessions or from a pharmacy-that a client received while in a facility. The intention of reporting services received from both observation and exit interview data is not to directly compare but to illustrate the relative frequency with which clients receive services. Following clients throughout their entire facility visit including interactions with auxiliary staff, such as pharmacy and laboratory personnel, would provide a more accurate picture of the services received, but cost and time restrictions did not allow for this.

\section{Conclusion}

This evaluation examines one major context in which PrEP and other novel prevention technologies are destined to be introduced. It helps to fill a gap in evidence on system strengthening necessary for moving PrEP and other ARV-based prevention technologies from clinical trial settings into real-world service delivery (37). For reasons of sustainability, acceptability and efficiency, the provision of these products will likely be through integrated and not stand-alone services. Yet, current challenges with the provision of integrated services raise the question of how burdened health systems are going to manage to incorporate new requirements into existing services (38). Governments and their partners must devise PrEP rollout strategies that acknowledge and address pre-existing supply side challenges.

\section{Declarations}

\section{Ethical approval}

This study was approved by the institutional review boards at FHI 360 and the Kenya Medical Research Institute (KEMRI). Each participant provided verbal informed consent prior to clinical observations and exit-interviews. 


\section{Consent for publication}

Not applicable.

\section{Availability of data and materials}

The datasets generated and analyzed during the current study are not publicly available as consent was not obtained from study participants to share the data publicly. Qualitative data are not available to avoid deductive disclosure of study participants.

\section{Competing interests}

The authors declare that they have no competing interests.

\section{Funding}

This work is made possible by the generous support of the American people through the U.S. Agency for International Development (USAID). Financial assistance was provided by USAID to FHI 360 under the terms of the Preventive Technologies Agreement No. GHO-A-00-09-00016-00. The contents do not necessarily reflect the views of USAID or the United States Government.

\section{Authors' contributions}

$\mathrm{EE}, \mathrm{TH}, \mathrm{ME}, \mathrm{AO}$ and $\mathrm{GG}$ conceptualized the study and designed collection of the data. $\mathrm{EE}, \mathrm{TH}, \mathrm{ME}, \mathrm{AO}$ and GG contributed to the acquisition of the data. EE, TH, ME and PO contributed to the analysis and interpretation of the data. $\mathrm{EE}, \mathrm{TH}, \mathrm{ME}$ drafted and revised the work. All authors have read and approved the submitted version and agree to be personally accountable for their own contributions.

\section{Acknowledgments}

The study team would like to thank the research supervisors, data manager, research assistants, and the county health directors of Mombasa, Nairobi, and Kisumu counties; the health facilities; and the health facility clients who participated in this study.

\section{References}

1. Caceres C, Mayer K, Baggaley R, O'Reilly K. PrEP Implementation Science: State-of-the-Art and Research Agenda. J Int AIDS Soc. 2015;18(4(Suppl 3)). 
2. Fonner V, Dalglish S, Kennedy C, Baggaley R, O'Reilly K, Koechlin F, et al. Effectiveness and safety of oral HIV preexposure prophylaxis for all populations AIDS. 2016;30.

3. WHO. WHO Expands recommendations on oral pre-exposure prophylasis of HIV infection (PrEP). Geneva, Switzerland 2015.

4. McCarten-Gibbs M, Allinder S. The evolution and future of HIV prevention technology. An HIV policy primer. Washington, DC: Center for Strategic and International Studies; 2019.

5. Zablotska I, Baeten J, Phanuphak N, McCormack S, Ong J. Gettubg pre-exposure prophylaxis (PrEP) to the people: opportunities, challenges and examples of successful health service models of PrEP implementation. Sex Health. 2018;15:481-4.

6. Dickinson C, Attawell K, Druce N. Progress on scaling up integrated services for sexual and reproductive health and HIV. Bull World Health Organ. 2009;87(11):846-51.

7. Gengiah T, Abdool Karim Q. Implementing microbicides in low income countries. Best Pract Res Clin Obstet Gynaecol. 2012;26(4):495-501.

8. Woodsong C, McGrory E, Farley T. Preparing for Microbicide Introduction, Rollout, and Sustained Access. Curr Top Microbiol Immunol. 2014(383):153-75.

9. Venter W, Cowan F, Black V, Rebe K, Bekker L. Pre-exposure prophylaxis in Southern Africa: feasible or not? J Int AIDS Soc. 2015;18((4Suppl 3)).

10. Bridget G, Glasgow R. Evaluation approaches for dissemination and implementation research. In: Brownson RC, Colditz GA, Proctor EK, editors. Dissemination and Implementation Research in Health Translating Science to Practice. New York: Oxford University Press; 2012.

11. Thrun M. Provider-Initiated HIV-Risk Behavior Counseling in the Context of HIV Pre-Exposure Prophylaxis. Am J Prev Med. 2013;44(1):S108-S11.

12. Underhill K, Operario D, Skeer M, Mimiaga M, Mayer K. Packaging PrEP to Prevent HIV: An Integrated Framework to Plan for Pre-Exposure Prophylaxis Implementation in Clinical Practice. J Acquir Immune Defic Syndr. 2010;55(1):8-13.

13. Underhill K, Operario D, Mimiaga M, Skeer M, al. e. Implementation science of pre-exposure prophylaxis: preparing for public use. Curr HIV/AIDS Rep. 2010;7(4):201-90.

14. Lanham M, Wilcher R, Montgomery E, Pool R, Schuler S, Lenzi R, et al. Engaging male partners in women's microbicide use: evidence from clinical trials and implications for future research and microbicide introduction. J Int AIDS Soc. 2014;17(Suppl 2).

15. Krakower D, Mayer K. Engaging healthcare providers to implement HIV pre-exposure prophylaxis. Curr Opin HIV AIDS. 2012;7(6):593-9.

16. Heise L, Shapiro K, West Slevin. Mapping the standard of care at microbicide clinical trial sites. Washington DC: Global Campaign for Microbicides; 2008.

17. Cowan F, Delany-Moretlwe S, Sanders E, Mugo N, Guedou F, Alary M, et al. PrEP implementation research in Africa: what is new? J Int AIDS Soc. 2016;19((Suppl6)). 
18. Sweeney S, Obure C, Maier C, Greener R, Dehne K, Vassall A. Costs and efficiency of integrating HIV/AIDS services with other health services: a systematic review of evidence and experience. Sex Transm Infect. 2012;88:85-99.

19. Celum C, Delany-Moretlwe S, MCConnell M, van Rooyen H, Bekker L, Kurth A, et al. Rethinking HIV prevention to prepare for oral PrEP implementation for young African Women. J Int AIDS Soc. 2015;18((4Suppl 3)).

20. Wilcher R, Hoke T, Adamchak SE, Cates W. Integration of family planning into HIV services: a synthesis of recent evidence. AIDS. 2013;27((Suppl 1)):S65-S76.

21. Dudley L, Garner P. Strategies for integrating primiary health services in low- and middle-income countries. Cochrane Database Syst Rev. 2014;7(CD003318).

22. Church K, Mayhew S. Integration of STI and HIV prevention, care and treatment into family planning services: a review of the literature. Stud Fam Plann. 2009;40:171-86.

23. Hoke T, Harries J, Crede S, Green M, al. e. Expanding contraceptive options for PMTCT clients: a mixed methods implementation study in Cape Town, South Africa. Repro Health. 2014;11(3).

24. Minimum package for reproductive health $(\mathrm{RH})$ \& HIV integrated services Nairobi, Kenya: Ministry of Health 2012.

25. Assessing Physical Delivery of PrEP in Support of Proof of Deliverability: Results from Kenya. Study Brief. FHI 360; 2013.

26. Mack N, Evens E, Tolley E, Brelsford K, Mackenzie C, Milford C. The importance of choice in the rollout of ARV-based prevention to user groups in Kenya and South Africa: a qualitative study. J Int AIDS Soc. 2014.

27. FHI 360. Assessing Physical Delivery of PreP in Support of Proof of Deliverability Study Briefs 2014 [cited 2014. Available from: http://www.fhi360.org/resource/assessing-physical-delivery-prepsupport-proof-deliverability-study-briefs.

28. Final results of FEM-PREP HIV-prevention study indicate great attention to adherence will be required in PrEP programs. Research Triangle Park, NC: FHI 360; 2012.

29. Guidelines for HIV Testing and Counselling in Kenya. Nairobi: National AIDS Control Programme Ministry of Public Health and Sanitation; 20102010.

30. Jenkinson K, Naughton G, Benson A. The GLAMA (Girls! Lead! Achieve! Mentor! Activate!) physical activity and peer leadership intervention pilot project: A process evaluation using the RE-AIM framework. BMC Public Health. 2012;12(1):55.

31. Brant A, Dhillon P, Hull S, Coleman M, Ye P, Lotke P, et al. Integration of HIV preexposure prophylaxis (PrEP) services with family planning services: an evaluation using the RE-AIM framework. Contraception 2018;98(4).

32. Kenya AIDS Response Progress Report 2014. Progress towards Zero; 2014.

33. Kenya AIDS Indicator Survey 2012: Final Report. Nairobi, Kenya: National AIDS and STI Control Programme (NASCOP); 2014. 
34. Mutemwa R, Mayhew S, Colombini, Busza J, Kivung J, Ndwiga. Experiences of health care providers with integrated HIV and reproductive health services in Kenya: a qualitative study. BMC Health Serv Res. 2013;13(18).

35. FHI 360. Microbicides Readiness Assessment Tool 2014 [Available from: http://www.fhi360.org/resource/microbicides-readiness-assessment-tool.

36. Everybody's business - strengthening health systems to improve health outcomes: WHO's framework for action. Geneva: World Health Organization; 2007.

37. Mugo N, Kenneth N, Michael K, Elizabeth I, Nduku K. The preexposure prophylaxis revolution; from clinical trials to programmatic implemenation. Curr Opin HIV AIDS. 2016;11(1):80 - 6.

38. Venter W. Pre-exposure prophylaxis: the delivery challenge. Front Public Health. 2018;6(6: 188).

\section{Figures}
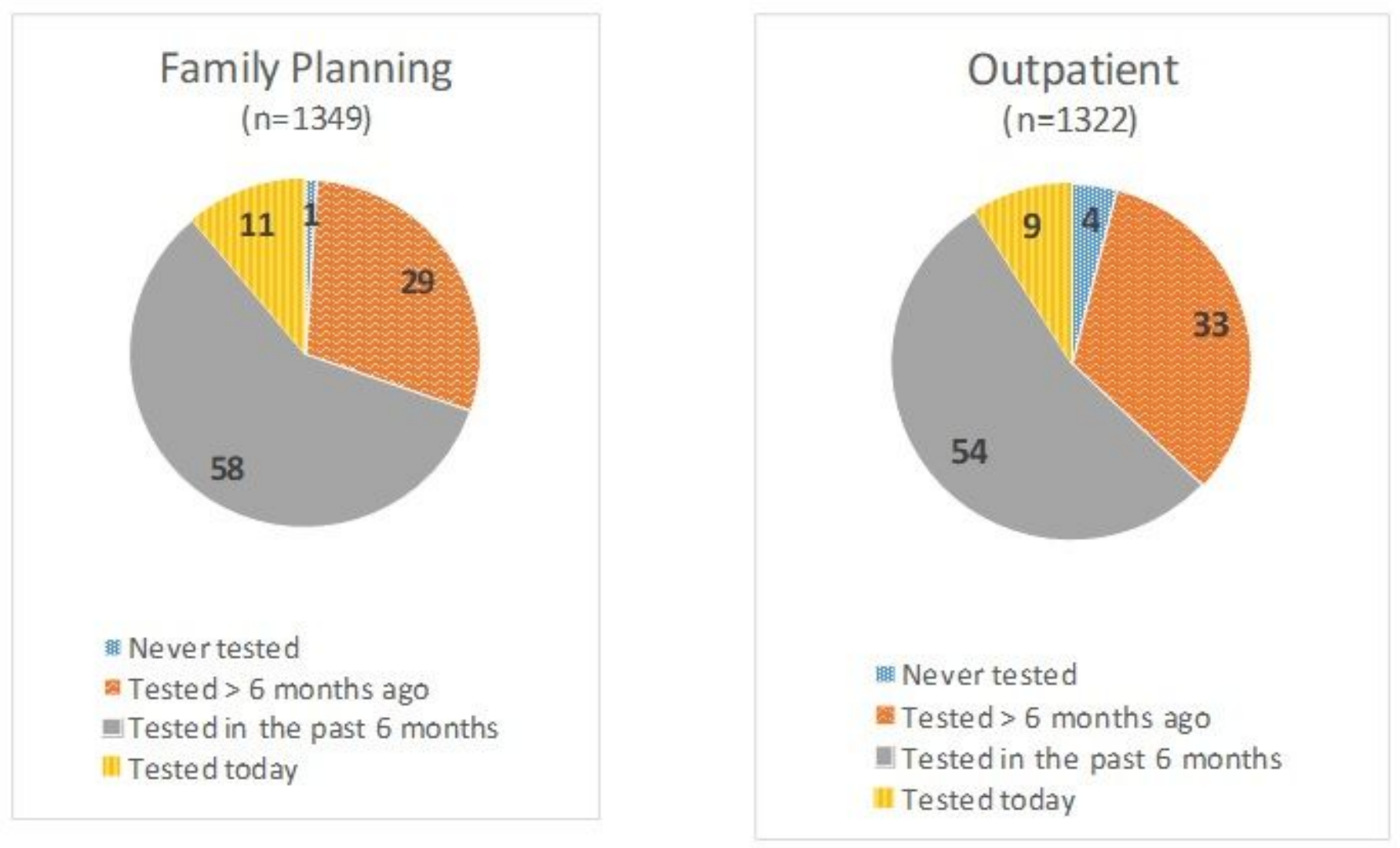

\section{Figure 1}

Time since last HIV test, by client type, from client exit interviews 